\title{
Optimum synthesis of steel plane trusses with subdivided panels
}

\author{
Igor Serpik ${ }^{1, *}$, and Bogdan Komshin ${ }^{1}$ \\ ${ }^{1}$ Bryansk State Engineering Technological University, Stanke Dimitrov av., 3, Bryansk, 241037, \\ Russia
}

\begin{abstract}
A computation scheme for topology and size optimization of steel trusses with subdivided panels has been developed. The framework strength, stiffness and stability limitations have been taken into consideration. The search for effective solutions is carried out on the basis of a genetic algorithm. An example of using the approach for optimization of the plane lenticular girder-type truss with $72 \mathrm{~m}$ span is given. A topology variant of the additional rods system for the truss panels and values of rod cross-sections for the whole structure has been obtained.
\end{abstract}

\section{Introduction}

It is civil engineering common practice to increase the steel truss load-carrying ability by subdividing panels using additional rods having relatively small cross-section areas. However, the issues of selecting topology and parameters of such systems have been understudied so far. The most effective direction in this field is the optimum design of structures. In this case, it is necessary to take into consideration a set of limitations allowing to ensure the strength, stiffness and stability of trusses.

Great attention is paid in scientific literature to the theory of truss structure optimization [1-3]. Many authors used for this purpose different metaheuristic computation schemes including genetic algorithms [4-10]. The procedures based on genetic operators allow solving extremum problems on discrete sets of variable topologies and parameters that corresponds to the real-life practice of structural design. This paper develops a procedure of genetic algorithm enabling the efficient search for system topology of the auxiliary rods subdividing panels, and cross-sections of the whole truss' rods. The efficiency of this method is illustrated by the example of optimal design of lenticular truss.

\section{Problem formulation}

It is supposed that topology of the main truss has been specified. Some redundant structure is introduced for the system of additional rods. Its control stipulates the possibility of setting "zero" (absent) rods by entering relatively low dummy modules elasticity of material. In this case, topological and parametric optimization is reduced to parametric.

Let us set the task to minimize the weight $M$ of the truss rods:

*Corresponding author: inserpik@gmail.com 


$$
M\left(L_{1}, L_{2}, \ldots, L_{n}\right) \rightarrow \min
$$

where $L_{i}(i=1,2, \ldots, n)$ are the discrete sets of allowable cross sections of rods, $n$ is the number of rods.

The possibility to vary the cross sections of only a part of rods is also provided. In addition, rods may be integrated into groups within each of which cross-sections are considered to be equal.

The stress-strain state of the load-carrying system is calculated by means of finiteelement method in the form of the slope deflection method [11]. The following limitations are taken into account:

A) Stability of geometrical shape of the structure.

B) Strength state condition:

$$
\omega_{\sigma}=\sigma_{M} / R-1 \leq 0
$$

where $\omega_{\sigma}$ is the parameter used to characterize the stress constraint satisfaction, $\sigma_{M}$ is the von Mises stress, $R$ is the allowable stress of steel set according to yield strength.

C) Providing the required stiffness:

$$
\omega_{\delta}=|\delta| / f-1 \leq 0
$$

where $\omega_{\delta}$ is the parameter used to characterize the filfilment of displacement limitations, $\delta$ is the vertical or horizontal displacement of the finite-element model node, $f$ is the allowable value for the considered displacement.

D) Providing the stability of rod system.

E) Condition of local stability of sheets of thin-walled profiles.

F) Providing local strength of the truss.

$G)$ Condition for the limiting slenderness ratio of rods.

H) Design and technological conditions.

\section{Optimization procedure}

It is deemed that A-D imitations are directly taken into consideration during optimization. Limitations $\mathrm{E}, \mathrm{G}$ and $\mathrm{H}$ are considered when specifying sets $L_{i}$, topology of the main loadcarrying system and redundant topology of the system of additional rods. Design solutions for complying with limitation $\mathrm{F}$ can be developed after optimization. It is assumed that the truss material is in linear-elastic stage. Tension-compression strain, flexural strain in two main planes and pure torsion strain are taken into consideration for the rods.

Conditions A and D will be reduced to displacement limitations. To that end, first of all, stability of geometrical shape of the redundant structure for initial shall be ensured. The operability analysis of the state of construction variants cross-sections of the whole truss' rods are carried out on the basis of strain state truss calculation using step-by-step method with due allowance for the rod bending effect caused by axial forces based on the use of geometrical matrices of finite elements [11]. The calculation of the load-carrying system without considering the bending effect caused by axial forces is implemented in the first iteration. In each iteration $r>1$ we solve the following simultaneous algebraic equations:

$$
\left([K]+\left[K_{G}\left(\{N\}^{(r-1)}\right)\right]\right)\{\delta\}^{(r)}=\{Q\},
$$

where $[K]$ is the stiffness matrix of the finite-element model, $\left[K_{G}\left(\{N\}^{(r-1)}\right)\right]$ is the geometric matrix of the finite-element system, expressed through the vector of longitudinal forces $\{N\}^{(r-1)}$ in rods found in iteration $r-1,\{\delta\}^{(r)}$ is the vector of nodal displacements 
calculated in iteration $r ;\{Q\}$ is the vector of reduced to nodes exterior loads taking into consideration the gravity force change due to variation of the structure parameters.

To provide the possibility of checking the stability on bulges from the structure plane, let us introduce a self-balancing system of small auxiliary couple of forces the planes of which are perpendicular to the truss plane. The calculations show that if a strained body of the framework becomes geometrically unchangeable, or its stability is not provided in terms of Euler's theory, it may be manifested by relatively large dummy displacements obtained in formal problem solution.

Approaches of [7] are used for constructing the selection, crossing and mutation for the genetic algorithm. Each discrete set of acceptable values of parameters is arranged from smallest to largest. In the evolutionary scheme we take into consideration the main population $W_{1}$ from $N$ projects and auxiliary population $W_{2}$ of improved projects the number of which depends on the evolutionary algorithm operation results, but does not exceed $N$. Initially we form the projects of population $W_{1}$ from identical construction variants with full redundant topology and maximum cross-section areas. Then the iteration process is executed. Each iteration of it includes the following basic operations:

1) Check for compliance with the active limitations for the projects of population $W_{1}$. Projects of this population are divided into subgroups $\alpha$ and $\beta$. If at least one limitation is not fulfilled for any of the subgroup $\alpha$ projects, then it is replaced with a project from population $W_{2}$ which is not used in the population $W_{1}$, or a newly formed variant of the load-carrying system. If the full compliance with such limitations is not achieved for the projects of subgroup $\beta$, then a penalty is introduced by multiplying the objective function value by a coefficient:

$$
k_{p}=\left(1+\gamma_{\sigma} \chi\left(\omega_{\sigma \max }\right) \omega_{\sigma \max }\right)\left(1+\gamma_{\delta} \chi\left(\omega_{\delta \max }\right) \omega_{\delta \max }\right)
$$

where $\gamma_{\sigma}, \gamma_{\delta}$ are the given positive numbers; $\chi(x)$ is the Heaviside function of some argument $x(\chi(x)=0$, if $x<0 ; \chi(x)=1$, if $x \geq 0) ; \omega_{\sigma \max }, \omega_{\delta \max }$ are maximum values of $\omega_{\sigma}, \omega_{\delta}$ for the checked construction variant in accordance with all the loads under consideration.

2) Adjustment of the population $W_{2}$ of improved projects. Each project of the population $W_{1}$ is checked according to two criteria: whether such construction variant is present in the population $W_{2}$ and whether the value $M$ of the variant doesn't exceed the maximum value of the objective function in that population. If both of these conditions are not fulfilled, then the project is included into the population $W_{2}$. If the number of construction variants in the auxiliary population exceeds $N$, the project with the maximum value $M$ is removed from it.

3) Execution of the mutation. During implementation of the mutation for the considered parameter, alternation of variants of its random change is provided both by selecting any element from a set of acceptable values, and only from values neighbouring to the current position of the parameter in that set.

4) An additional check for compliance with limitations for the projects of population $W_{1}$ and repeated editing of population $W_{2}$.

5) Selection based on the roulette method and single-point crossover for the individuals of population $W_{1}$.

The calculations show that in case of the optimum synthesis of structures of this type using the presented evolutionary scheme, any absence of changes in the population $W_{2}$ during 200-250 iterations means it is expedient to stop the optimization. Any further continuation of the iteration process does not usually result in any significant change of parameters for the most rational projects. For the final design solution an additional adjusted check for compliance with the set limitations shall be carried out. 


\section{Example of optimum design}

Let us illustrate the possibilities of the approach being developed on the example of a steel roof truss optimization. The truss with $72 \mathrm{~m}$ span and $9 \mathrm{~m}$ height is made according to the girder scheme and is lenticular. Figure 1 illustrates the main truss, useful load in the form of forces $P=120 \mathrm{kN}$ transferred from the longitudinal girders and external constraints of the structure.

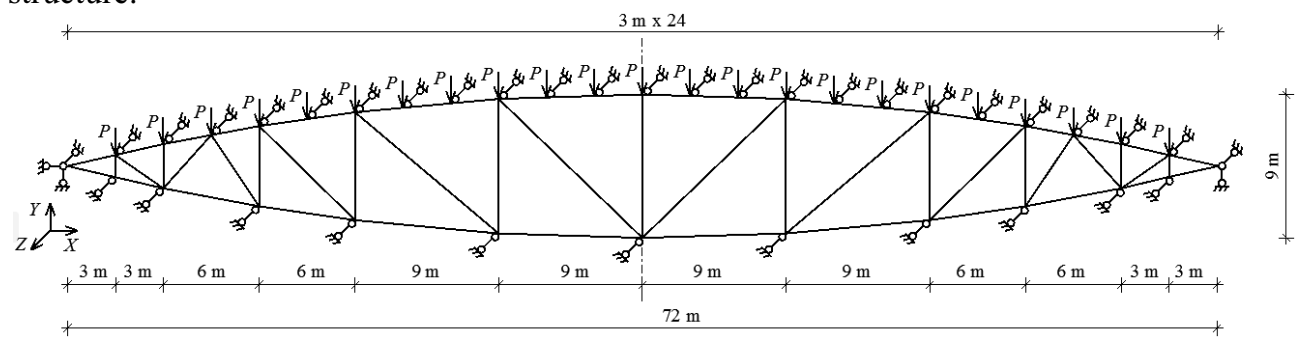

Fig. 1. Main truss.

A redundant topology of additional rods shown in Fig. 2 was introduced. Conceptually, such a topology allows taking up forces $P$ in the truss joints and increasing the stability of compressed rods of the main truss.

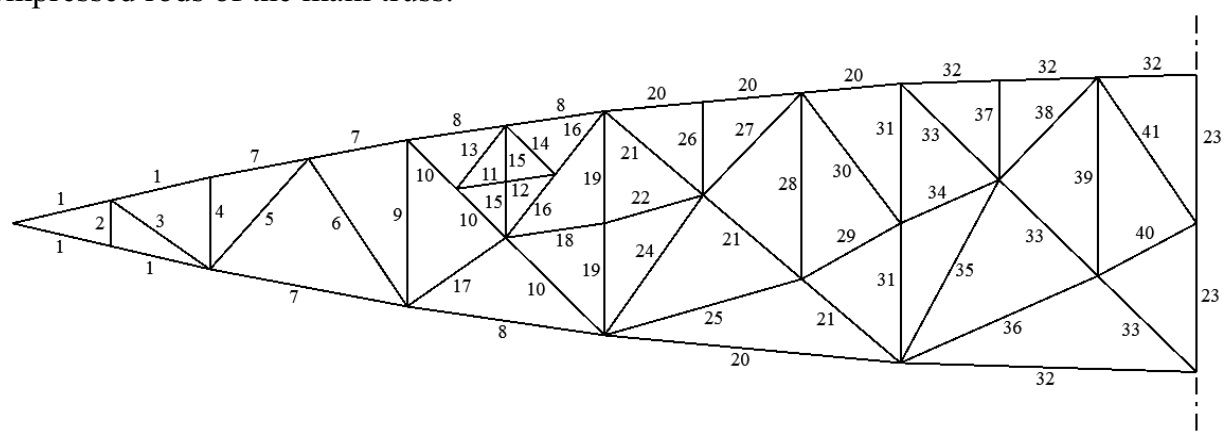

Fig. 2. Redundant topology for a half of the symmetrical structure with reference to the rod group numbers.

Steel S245 (Russian construction regulations SP 16.13330.2011. Steel Structures) was specified as a material for all rods. It was assumed that the truss rods were made of thinwalled rectangular pipes (Fig. 3). In this case $y$ axis is perpendicular to the truss plane. Totally 41 rod groups were used. The rod grouping for a half of the symmetrical structure is shown in Fig. 2. The allowable cross-sections sizes according to GOST 30245-2012 (Steel bent closed welded square and rectangular section for building. Specifications) for the truss rod groups are specified in Tables 1-4, where $N_{S}$ is the number of cross-section, $h, b$ are the cross-section height and width; $t$ is the wall thickness. 


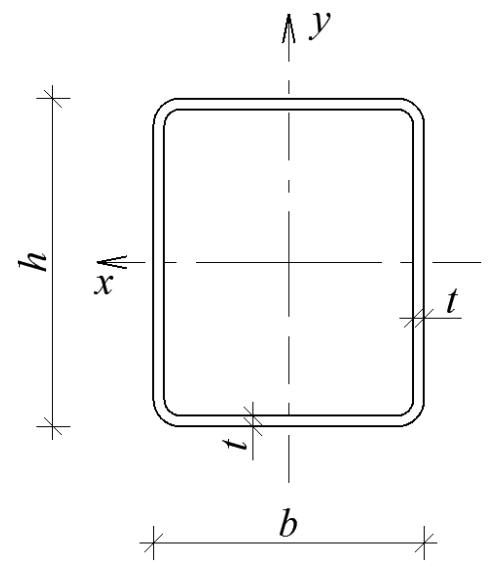

Fig. 3. Cross-section of truss rods.

The load-carrying ability limitations were assumed in accordance with SP 16.13330.2011. When calculating the stress-strain state of the structure, each rod was divided into 6 finite elements. Optimization results are presented in Fig. 4 and in Table 5.

Table 1.Allowable cross-sections for rod groups 1, 7, 8, 20, 32.

\begin{tabular}{|c|c|c|c|}
\hline$N_{s}$ & $\begin{array}{c}h, \\
\text { mm }\end{array}$ & $\begin{array}{c}b, \\
\text { mm }\end{array}$ & $\underset{\mathbf{m m}}{t,}$ \\
\hline 1 & \multirow[t]{28}{*}{500} & \multirow[t]{28}{*}{300} & 7 \\
\hline 2 & & & 7.5 \\
\hline 3 & & & 8 \\
\hline 4 & & & 8.5 \\
\hline 5 & & & 9 \\
\hline 6 & & & 9.5 \\
\hline 7 & & & 10 \\
\hline 8 & & & 10.5 \\
\hline 9 & & & 11 \\
\hline 10 & & & 11.5 \\
\hline 11 & & & 12 \\
\hline 12 & & & 12.5 \\
\hline 13 & & & 13 \\
\hline 14 & & & 13.5 \\
\hline 15 & & & 14 \\
\hline 16 & & & 14.5 \\
\hline 17 & & & 15 \\
\hline 18 & & & 15.5 \\
\hline 19 & & & 16 \\
\hline 20 & & & 16.5 \\
\hline 21 & & & 17 \\
\hline 22 & & & 17.5 \\
\hline 23 & & & 18 \\
\hline 24 & & & 18.5 \\
\hline 25 & & & 19 \\
\hline 26 & & & 20 \\
\hline 27 & & & 21 \\
\hline 28 & & & 22 \\
\hline
\end{tabular}


Table 2. Allowable cross-sections for rod groups 2-6, 9, 11-18, 22-30, 34-41.

\begin{tabular}{|c|c|c|}
\hline $\boldsymbol{N}_{\boldsymbol{s}}$ & $\boldsymbol{h}=\boldsymbol{b}, \mathbf{m m}$ & $\boldsymbol{t}, \mathbf{m m}$ \\
\hline 1 & 100 & 3 \\
\hline 2 & 120 & 3 \\
\hline 3 & 140 & 4 \\
\hline 4 & 150 & 4 \\
\hline 5 & 160 & 4 \\
\hline 6 & 180 & 5 \\
\hline
\end{tabular}

Table 3. Allowable cross-sections for rod groups 10, 19, 23, 31.

\begin{tabular}{|c|c|c|c|}
\hline $\boldsymbol{N}_{\boldsymbol{s}}$ & $\boldsymbol{h} \mathbf{\mathbf { m m }}$ & $\boldsymbol{b}, \mathbf{m m}$ & $\boldsymbol{t}, \mathbf{m m}$ \\
\hline 1 & 120 & 60 & 3 \\
\hline 2 & 160 & 80 & 4 \\
\hline 3 & 180 & 100 & 4 \\
\hline 4 & 200 & 100 & 4 \\
\hline 5 & 240 & 120 & 5 \\
\hline 6 & 260 & 130 & 6 \\
\hline
\end{tabular}

Table 4. Allowable cross-sections for rod groups 21, 33.

\begin{tabular}{|c|c|c|c|}
\hline $\boldsymbol{N}_{\boldsymbol{s}}$ & $\boldsymbol{h} \mathbf{\mathbf { m m }}$ & $\boldsymbol{b}, \mathbf{m m}$ & $\boldsymbol{t}, \mathbf{~ m m}$ \\
\hline 1 & 180 & 60 & 4 \\
\hline 2 & 200 & 80 & 4 \\
\hline 3 & 180 & 120 & 4 \\
\hline 4 & 200 & 120 & 4 \\
\hline 5 & 220 & 140 & 5 \\
\hline 6 & 240 & 160 & 5 \\
\hline 7 & 300 & 100 & 6 \\
\hline 8 & 300 & 200 & 6 \\
\hline
\end{tabular}

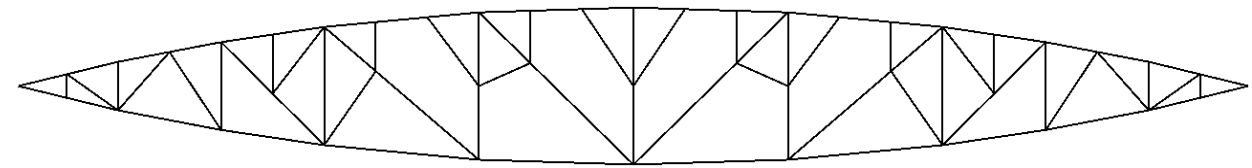

Fig. 4. Result of topology optimization.

Table 5. Result of selection of cross-sections.

\begin{tabular}{|c|c|c|c|}
\hline Rod group numbers & $\boldsymbol{h}, \mathbf{m m}$ & $\boldsymbol{b}, \mathbf{m m}$ & $\boldsymbol{t}, \mathbf{m m}$ \\
\hline 1 & 500 & 300 & 13 \\
\hline $2-6,9,11-18,22-30,34-41$ & 100 & 100 & 3 \\
\hline 7,8 & 500 & 300 & 11 \\
\hline $10,19,23,31$ & 120 & 60 & 3 \\
\hline 21,33 & 180 & 60 & 4 \\
\hline 32 & 500 & 300 & 10.5 \\
\hline
\end{tabular}

Fig. 5 illustrates the graph of change of the objective function in the evolutionary process. By iteration 202 the truss weight amounted $21,420 \mathrm{~kg}$, and it was not adjusted till iteration 701. The checking calculations of this construction variant based on the finite element model have shown that all specified requirements in terms of strength, stiffness and stability are met for it. 


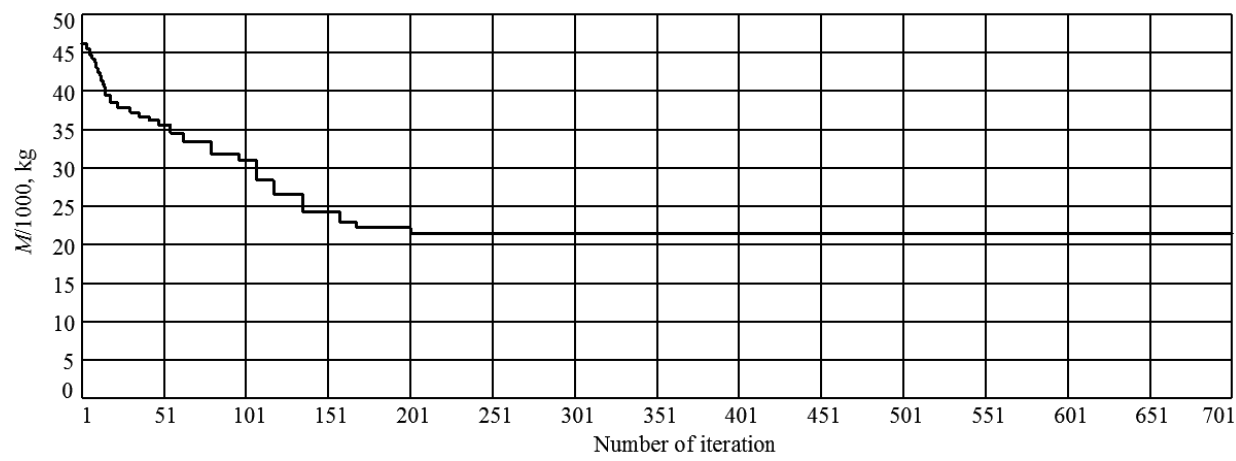

Fig 5. Dependence of truss rod weight from the number of iteration.

\section{Conclusion}

The methodology for optimum design of steel plane truss structures where the main truss panels are subdivided by additional rods has been developed. A computation scheme using the methodology of redundant topologies and genetic operators was formulated for designing such type of objects taking into account active limitations on rod strength, displacements and stability of the framework. The limitations on walls of rods stability are taken into account for assigning allowable variants of their cross-sections. The suggested approach allows selecting a topology of the auxiliary rods and carrying out a discrete search for cross-sections for all truss rods. The efficiency of the presented algorithm is illustrated by the example of optimization of steel lenticular truss.

\section{References}

1. S. Šilih, S. Kravanja, WIT Transactions on the Built Environment, 80, 221-333 (2005)

2. A.S. Marutyan, Structural Mechanics and Analysis of Constructions, 2(253), 60-66 (2014)

3. V.A. Zinkova,Bulletin of Belgorod State Technological University (BSTU) named after V.G. Shukhov, 2, 37-40 (2015)

4. F. Flager, G. Soremekun, A. Adya, K. Shea, J. Haymaker, M. Fischer, Computers and Structures, 140, 55-65 (2014)

5. M.S. Goncalves, R.H. Lopez, L.F.F. Miguel, Computers and Structures, 153, 165-184 (2015)

6. A.G. Yuryev, S.V. Klyuyev, Evolutionary and genetic algorithm of optimization of building structures (Belgorod State Technological University, Belgorod, 2006)

7. I.N. Serpik, A.V. Alekseytsev, Structural Mechanics and Analysis of Constructions, 5, 58-63 (2011)

8. M.H. Talebpour, A. Kaveh, V.R. Kalatjari, Iranian Journal of Science and Technology, Transactions of Civil Engineering, 38, 1-20 (2014)

9. I.N. Serpik, A.V. Alekseytsev, Magazine of Civil Engineering, 1(61), 14-24 (2016)

10. I.N. Serpik, I.V. Mironenko, V.I. Averchenkov, Procedia Engineering, 150, 1311-1316 (2016)

11. O.C. Zienkiewicz, R.L. Taylor, D. Fox, The finite element method for solid and structural mechanics (Elsevier, Oxford, 2014) 